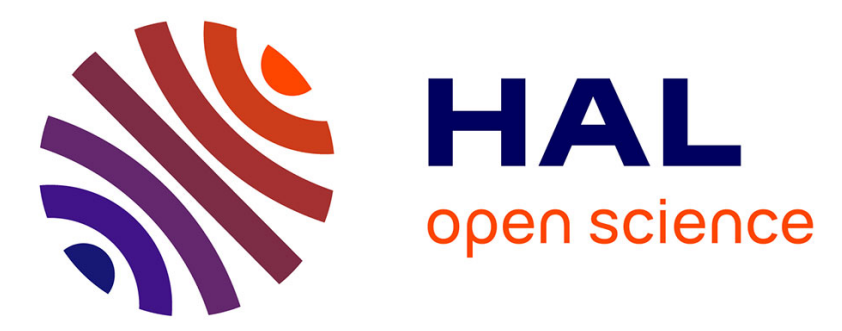

\title{
A Global Shape Index to Characterize Anterior Lamina Cribrosa Morphology and its Determinants in Healthy Indian Eyes
}

Sri Gowtham Thakku, Yih-Chung Tham, Mani Baskaran, Jean-Martial Mari, Nicholas G Strouthidis, Tin P Aung, Ching-Yu Cheng, Michael J A Girard

\section{To cite this version:}

Sri Gowtham Thakku, Yih-Chung Tham, Mani Baskaran, Jean-Martial Mari, Nicholas G Strouthidis, et al.. A Global Shape Index to Characterize Anterior Lamina Cribrosa Morphology and its Determinants in Healthy Indian Eyes. Investigative Ophthalmology \& Visual Science, 2015, 56 (6), p. 3604 3614. 10.1167/iovs.15-16707 . hal-01711217

\section{HAL Id: hal-01711217 \\ https://hal.science/hal-01711217}

Submitted on 16 Feb 2018

HAL is a multi-disciplinary open access archive for the deposit and dissemination of scientific research documents, whether they are published or not. The documents may come from teaching and research institutions in France or abroad, or from public or private research centers.
L'archive ouverte pluridisciplinaire HAL, est destinée au dépôt et à la diffusion de documents scientifiques de niveau recherche, publiés ou non, émanant des établissements d'enseignement et de recherche français ou étrangers, des laboratoires publics ou privés. 


\title{
A Global Shape Index to Characterize Anterior Lamina Cribrosa Morphology and Its Determinants in Healthy Indian Eyes
}

\author{
Sri Gowtham Thakku, ${ }^{1}$ Yih-Chung Tham, ${ }^{1,2}$ Mani Baskaran, ${ }^{1-3}$ Jean-Martial Mari, ${ }^{4}$ \\ Nicholas G. Strouthidis, ${ }^{1,5,6}$ Tin Aung, ${ }^{1-3}$ Ching-Yu Cheng, ${ }^{1-3}$ and Michael J. A. Girard ${ }^{7,1}$ \\ ${ }^{1}$ Singapore Eye Research Institute, Singapore National Eye Centre, Singapore \\ ${ }^{2}$ Department of Ophthalmology, Yong Loo Lin School of Medicine, National University of Singapore, Singapore \\ ${ }^{3}$ Duke-National University of Singapore Graduate Medical School, Singapore \\ ${ }^{4}$ University of French Polynesia, Tahiti, French Polynesia \\ ${ }^{5}$ National Institute for Health Research Biomedical Research Centre, Moorfields Eye Hospital National Health Service Foundation \\ Trust \& University College London Institute of Ophthalmology, London, United Kingdom \\ ${ }^{6}$ Discipline of Clinical Ophthalmology and Eye Health, University of Sydney, Sydney, New South Wales, Australia \\ ${ }^{7}$ Department of Biomedical Engineering, National University of Singapore, Singapore
}

Correspondence: Michael J. A. Girard, In Vivo Biomechanics Laboratory, Department of Biomedical Engineering, National University of Singapore, 9 Engineering Drive 1 EA\#03-12, 117576, Singapore; mgirard@nus.edu.sg.

C-YC and MJAG contributed equally to the work presented here and should therefore be regarded as equivalent authors.

Submitted: February 18, 2015

Accepted: April 20, 2015

Citation: Thakku SG, Tham Y-C, Baskaran M, et al. A global shape index to characterize anterior lamina cribrosa morphology and its determinants in healthy Indian eyes. Invest Ophthalmol Vis Sci. 2015;56:3604-3614. DOI:10.1167/iovs.15-16707
Purpose. Lamina cribrosa (LC) morphology could be implicated in the progression of glaucoma. To date, no established, quantifiable parameter to assess LC shape in vivo exists. We aim to introduce a new global shape index for the anterior LC (LC-GSI) and to identify associations with ocular factors in a healthy Indian population.

Methods. Optical coherence tomography (OCT) scans of the optic nerve head (ONH) were performed on 162 healthy subjects. Optic nerve head structures were delineated and a geometric characterization of anterior LC morphology was obtained by measuring curvature along 180 LC cross sections and representing it as LC-GSI ranging from -1 to +1 . Lamina cribrosa depth and curvature were also reported. Linear regression was used to identify factors associated with LC morphology.

REsults. The typical healthy LC had a saddle rut-like appearance, with a central ridge visible in superior-inferior cross sections. A more prominent central ridge (larger LC-GSI) was associated with shorter axial length $(P<0.001)$, smaller Bruch's membrane opening (BMO) area $(P=0.020)$, smaller vertical cup-to-disc ratio $(\mathrm{VCDR})(P=0.007)$, and larger minimum rim width (BMO-MRW) $(P=0.001)$. A deeper LC was associated with male sex $(P<0.001)$, shorter axial length $(P=0.003)$, larger VCDR $(P<0.001)$, and smaller BMOMRW $(P=0.002)$. Age and IOP were not significantly associated with LC morphology in healthy eyes.

Conclusions. The LC-GSI is a single index that quantifies overall LC shape in an intuitive way. Ocular determinants of LC-GSI in healthy eyes included risk factors for glaucoma (axial length, VCDR, and BMO-MRW), highlighting the potential role of LC morphological characterization in the diagnosis and monitoring of glaucoma.

Keywords: lamina cribrosa morphology, optic nerve head, optical coherence tomography

\begin{abstract}
A major site of retinal ganglion cell (RGC) damage in A glaucoma is at the optic nerve head (ONH), where RGC axons congregate before making their way to the brain. ${ }^{1-3}$ Within the ONH, RGC axons pass through a mesh-like collagen fiber structure called the lamina cribrosa (LC), before exiting the eye.

Lamina cribrosa morphology and progression of glaucoma are believed to be closely related. ${ }^{4}$ In particular, glaucomatous onset is associated with posterior displacement of the anterior LC surface, ${ }^{5-7}$ posterior migration of the LC insertion points, ${ }^{6}$ and backward bowing of the entire LC. ${ }^{8}$ Nonhuman primate models of glaucoma have also shown similar associations. ${ }^{9-11}$ Furthermore, these models suggest that LC changes precede surface level retinal nerve fiber layer (RNFL) thinning, ${ }^{11,12}$ which is a useful marker of glaucoma onset. Taken together,
\end{abstract}

these findings indicate that an assessment of LC morphology may potentially be used as a biomarker for early glaucoma detection. The in vivo characterization of LC morphology in a healthy population is essential in this regard.

Previous studies have reported the variation of LC depth among healthy and glaucomatous eyes using in vivo spectraldomain optical coherence tomography (SD-OCT) imaging. ${ }^{13,14}$ Park et al. ${ }^{13}$ reported the presence of a central ridge in the LC and lower insertion points in the superior-inferior (S-I) cross section. Other studies have also shown the LC to be U- or Wshaped. 5,15-17 These findings, however, do not provide a quantitative measure of LC shape, which may be useful in detecting morphological changes. Moreover, most LC measurements in these studies looked at position, rather than shape, and were defined with reference to the Bruch's membrane 
opening (BMO). ${ }^{12,18,19}$ A recent study has shown axial variations in position of the $\mathrm{BMO}$ with age and race, ${ }^{20}$ placing limitations on measurements referenced to this landmark. While more advanced shape analysis techniques have been proposed, ${ }^{21,22}$ restricted LC visibility in OCT images limits their applicability to the characterization of human eyes in vivo.

In this study, we use image enhancement techniques to improve LC visibility in OCT images, delineate and create a three-dimensional (3D) reconstruction of the entire anterior LC, and introduce a global shape index (LC-GSI) to quantitatively measure overall anterior LC morphology. We demonstrate its robustness through repeatability analysis, compare it with existing LC parameters, and identify associated ocular factors in a healthy Indian population, a group that has not been studied previously.

\section{SubJeCts AND Methods}

\section{Study Subjects}

In this cross-sectional study, 223 consecutive subjects were prospectively recruited from the Singapore Indian Eye Study (SINDI), an ongoing population-based study at the Singapore Eye Research Institute (SERI). ${ }^{23}$

Each subject underwent an interview and ocular examinations according to a standardized study protocol, including imaging of the ONH region using SD-OCT (Spectralis SD-OCT; Heidelberg Engineering GmbH, Heidelberg, Germany), measurement of intraocular pressure (IOP) using Goldmann applanation tonometry (Haag-Streit, Bern, Switzerland), vertical cup-to-disc ratio (VCDR) from slit-lamp biomicroscopy using a graticule (Haag-Streit), central corneal thickness (CCT) using ultrasound pachymetry (Mentor O\&O, Inc., Norwell, MA, USA), axial length using noncontact partial coherence interferometry (Carl Zeiss Meditec AG, Jena, Germany), and corneal curvature using an autorefractor (Canon, Inc., Tokyo, Japan).

The study protocol was approved by the SingHealth Centralized Institutional Review Board and adhered to the ethical principles outlined in the Declaration of Helsinki, 2008. Written voluntary informed consent was obtained from each subject.

\section{Clinical Exclusion Criteria}

Subjects with a logarithmic minimum angle of resolution ( $\log$ MAR) visual acuity worse than 0.5 , evidence of retinopathy, previous ocular surgery (except cataract surgery), and neurological diseases and clinical features compatible with a diagnosis of glaucoma (suspect or manifest) were excluded from the study. Glaucoma suspect was defined as having any of the following criteria in the presence of normal visual field: IOP $>21 \mathrm{~mm} \mathrm{Hg}, \mathrm{VCDR}>0.7$, signs consistent with pseudoexfoliation or pigment dispersion syndrome, narrow angles (posterior trabecular meshwork visible for $<180^{\circ}$ during static gonioscopy), and peripheral anterior synechiae or other findings consistent with secondary glaucoma.

\section{OCT Image Acquisition}

Optical coherence tomography raster scans (with enhanced depth imaging or $\mathrm{EDI}^{24-28}$ ) were acquired for both ONHs of each subject. Each set of images comprised 72 serial horizontal B-scans (each composed of 384 A-scans) covering a rectangular region of $15^{\circ} \times 15^{\circ}$ centered on the ONH. Distance between consecutive scans varied slightly across different eyes, with a mean of $61.4 \pm 6.7 \mu \mathrm{m}$. The lateral resolution of each B-scan was $11.6 \pm 1.0 \mu \mathrm{m}$ horizontally and $3.9 \pm 0.0 \mu \mathrm{m}$ axially. To reduce speckle noise, each B-scan was averaged 20 times during acquisition.

\section{Image Delineation}

Optic nerve head structures in one randomly selected eye of each subject were delineated for further analysis. Selection was based on a random number generator on MATLAB (Mathworks, Inc., Natick, MA, USA). Raw OCT images were first enhanced using adaptive compensation to improve LC visibility. Adaptive compensation is an OCT postprocessing technique that removes shadows and enhances contrast. It has been shown to significantly improve visibility of the anterior LC, its insertions, and other ocular structures ${ }^{29-32}$ (Figs. 1A, 1B). Enhanced images were then loaded on a custom-written application on MATLAB that enabled 3D visualization and delineation.

Three ONH structures were delineated (Fig. 1C): the anterior LC surface, BMO, and the inner limiting membrane (ILM). The posterior LC surface was not delineated due to its consistently poor visibility. ${ }^{32}$

The position of the anterior LC was defined by a sharp increase in axial signal intensity below the optic disc, extending laterally up to the LC insertion points in the peripapillary sclera. ${ }^{33}$ Points corresponding to the LC were manually marked on each B-scan using our custom application. In total, 150 to 300 discrete points were marked for each eye. Regions of the LC that were deemed not visible were left unmarked.

In addition to the anterior $\mathrm{LC}$, the $\mathrm{BMO}$, defined as the point of termination of the Bruch's membrane layer, was marked using two discrete points on either side of the optic disc on each B-scan when visible (Fig. 1C). The ILM was automatically delineated by the Spectralis OCT device.

\section{D Reconstruction}

After delineation of the anterior LC, BMO, and ILM, the ONH structures were reconstructed in $3 \mathrm{D}$ in order to characterize LC morphology (Fig. 1D). A smoothing spline was used to generate the best-fitting surface corresponding to the delineated LC points. The advantages of fitting a surface are 2-fold. First, it reduces random variations that may have arisen due to speckle noise. Second, the morphology measurements performed on surfaces are more representative when compared with those using only the delineated points. Ren et al. ${ }^{7}$ explained the need to "weight" delineated points in order to compensate for variations in marking density across the lamina. Fitting a surface effectively resamples the delineated markings and creates points that are uniformly spaced, thereby overcoming this problem.

The BMO was reconstructed by first identifying the bestfitting plane to the delineated BMO points (BMO plane), and then fitting an ellipse to the projections of the BMO points on this plane (Figs. 2A, 2B). This strategy was similar to that in a previous study. ${ }^{33}$

In order to ensure consistency in measurements across different eyes, a common reference system was used. To that end, the BMO planes and centers (now taken as origin) of all patients were superimposed (Figs. 2C, 2D).

\section{Exclusion Criteria From 3D Reconstruction}

After delineation and reconstruction, eyes with extensive peripapillary atrophy (resulting in an incorrectly marked BMO) or a poorly visible LC (LC covering less than $70 \%$ of the BMO area from en face visualization) were excluded from the analysis (Figs. 3A-C). 

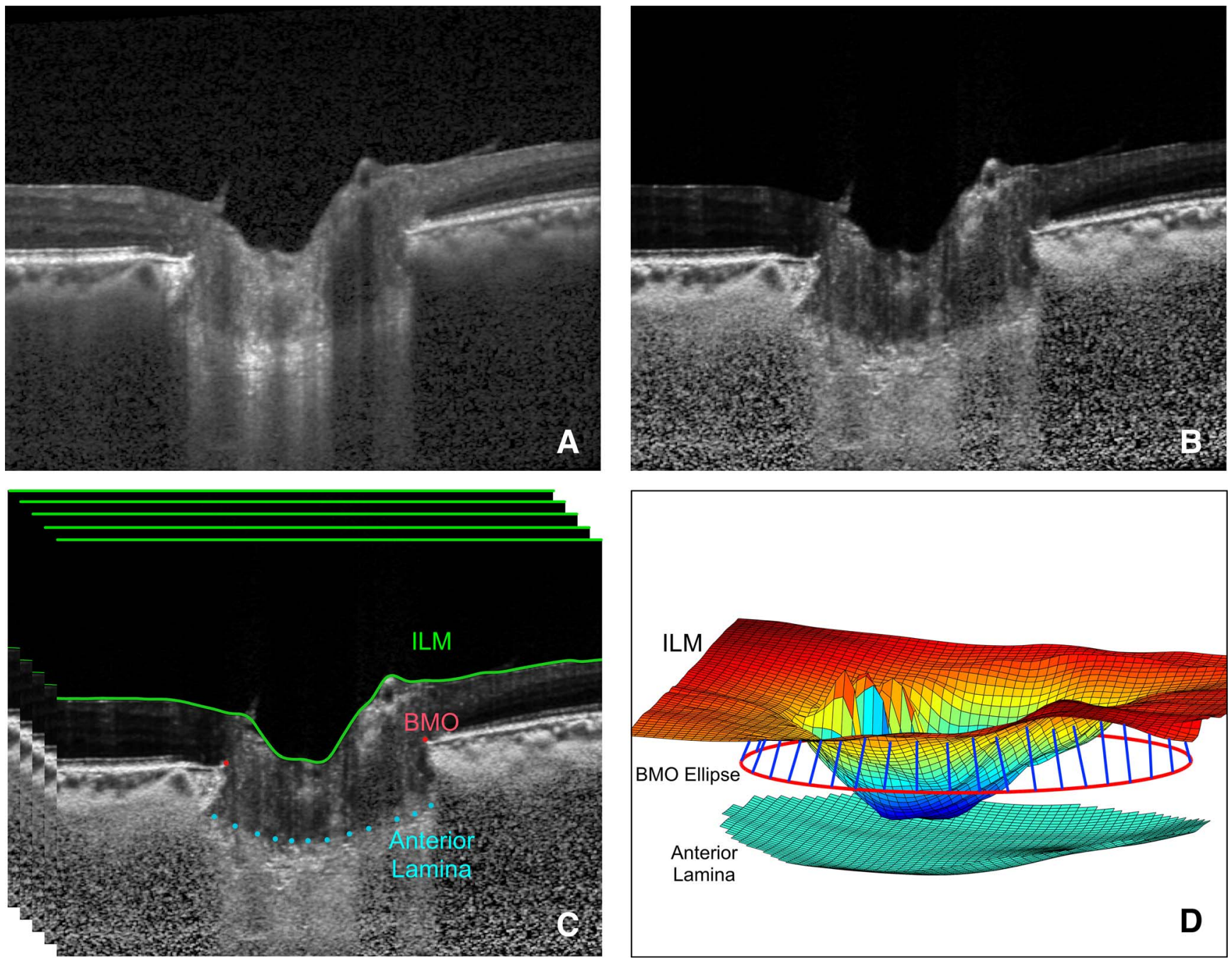

Figure 1. B-scan delineation of ONH structures and 3D reconstruction. (A) Original EDI-OCT image obtained from the Spectralis OCT device; (B) enhanced image with improved lamina visibility after application of adaptive compensation; (C) successive B-scans with delineated ILM, BMO, and LC; (D) B-scan delineations combined to generate 3D visualization; blue lines indicate minimum rim width.

\section{Measurement of Morphological Parameters}

We defined and calculated (in MATLAB) the following LC morphological parameters.

LC Depth. Lamina cribrosa depth was defined as the distance of the reconstructed anterior LC from BMO plane. The mean depth of all points on the surface was reported as the mean LC depth. Additionally, mean depths of points along the nasal-temporal (N-T) and superior-inferior (S-I) cross sections were reported.

LC Curvature. To measure LC curvature, we first intersected the anterior LC surface with 180 radial cross sections that passed through the center of the BMO ellipse and that were perpendicular to the BMO plane (Fig. 4A). Such a process generated 180 LC curves (one for each cross section). A circular arc was then fit to each LC curve (Figs. 4B, 4C, 5). Traditional methods for measuring curvature ${ }^{22}$ are extremely sensitive to local variations due to noise, but our relatively simple approach allowed us to estimate LC curvature along different radial orientations in a global sense (from fitted arcs) without compromising on robustness. A positive arc curvature indicates that the LC cross section is curved anteriorly (Fig. $4 \mathrm{D})$, whereas a negative arc curvature indicates that it is curved posteriorly (Fig. 4E). Near-zero arc curvature indicates a flatter cross section.

Lamina Cribrosa Global Shape Index. In order to provide a global measure of LC shape, we defined LC-GSI as ${ }^{34}$ :

$$
L C-G S I=\frac{2}{\pi} \tan ^{-1} \frac{\kappa_{1}+\kappa_{2}}{\kappa_{1}-\kappa_{2}}\left(\kappa_{1} \geq \kappa_{2}\right)
$$

where $\kappa_{1}$ and $\kappa_{2}$ are the maximum and minimum principal arc curvatures of the LC, respectively. The LC-GSI (based on Koenderink and Vandoorn ${ }^{34}$ ) is a number that varies between -1 and 1 , where -1 indicates a spherical cup (posteriorly curved LC), 0 a symmetric saddle-shaped LC, and 1 a spherical cap (anteriorly curved LC). These and other intermediate shapes are illustrated in Figure 6A. The actual LC morphologies associated with the range of LC-GSI values are depicted in Figure 5.

Mean Minimum Rim Width. In addition to the LC morphological parameters, the mean minimum rim width from the BMO (BMO-MRW) was measured, as it has recently been suggested to be an anatomically accurate biomarker for glaucoma. ${ }^{35}$ The mean shortest $3 \mathrm{D}$ distance from the BMO to the ILM (previous studies were in $2 \mathrm{D}^{35,36}$ ) was measured using custom code and reported as BMO-MRW (Fig. 1D). 

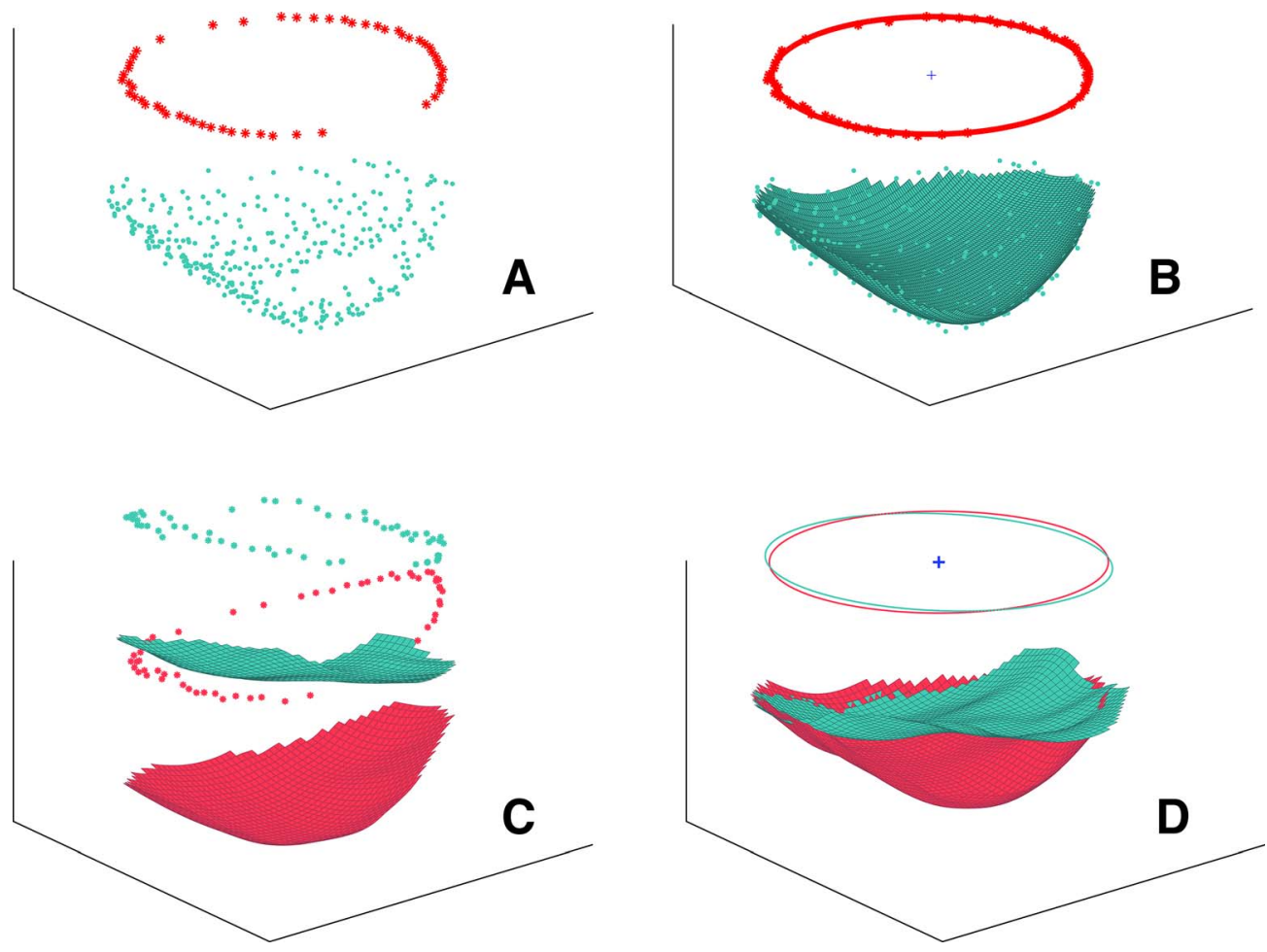

FigURE 2. 3D reconstruction from raw delineated points and definition of common reference system. (A) Raw markings of LC (cyan) and BMO (red) generated from delineated B-scans; (B) best-fitting surface to LC points and ellipse to BMO points superimposed on original points; (C) two reconstructed LCs in their original coordinate system acquired during imaging; (D) the same LCs displayed in the new coordinate system with the $\mathrm{BMO}$ planes aligned horizontally and the center of the BMO ellipse as the origin.

\section{Validation}

Validation involved demonstrating the robustness of our measurements. First, we evaluated the ability of our newly introduced parameter, LC-GSI, to adequately describe anterior LC shape. Second, we performed intraobserver and intra- and intervisit repeatability tests on a subset of randomly chosen eyes to determine the reliability of our measurements.

Evaluation of LC-GSI as a LC Shape Measure. The LCGSI is a single parameter aimed at describing global anterior LC shape. It is, however, derived from only two LC arc curvature values (among 180) and could thus be limited. To assess whether LC-GSI is in fact representative of the entire anterior
LC shape, the following analysis was performed. First, all the delineated LCs were assigned to corresponding predicted shapes based on their measured LC-GSI (Fig. 5). Second, for each LC, arc curvature was estimated for each of the 180 cross sections. Third, predicted (from LC-GSI) and estimated curvatures were compared using root mean square (RMS) errors. This approach can assess the accuracy of LC-GSI in describing anterior LC shape.

Measurement Repeatability. To assess the reliability of measurements based on manual delineations, a subset of eyes was randomly chosen and delineated twice by the same observer (SGT) 1 month apart. Intraobserver repeatability was performed on 50 eyes, and intraclass correlation coefficient
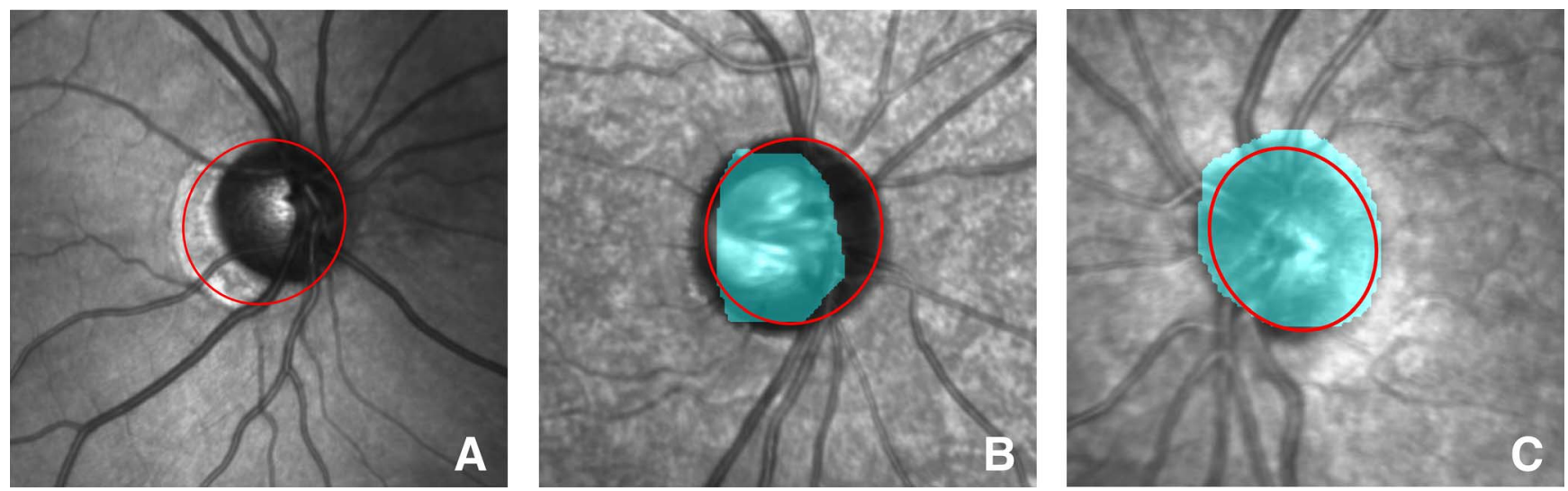

Figure 3. Delineated Bruch's membrane opening (red) and anterior lamina cribrosa (cyan) projected onto an en face image of the optic disc. (A) Eyes with peripapillary atrophy (PPA) were excluded due to potentially unclear BMO leading to overestimation of disc size; (B) eyes in which the LC spans less than $70 \%$ of the BMO were excluded from analysis (here 62\%); (C) eyes in which the LC spans greater than $70 \%$ of the BMO were included (here $100 \%$ ) 


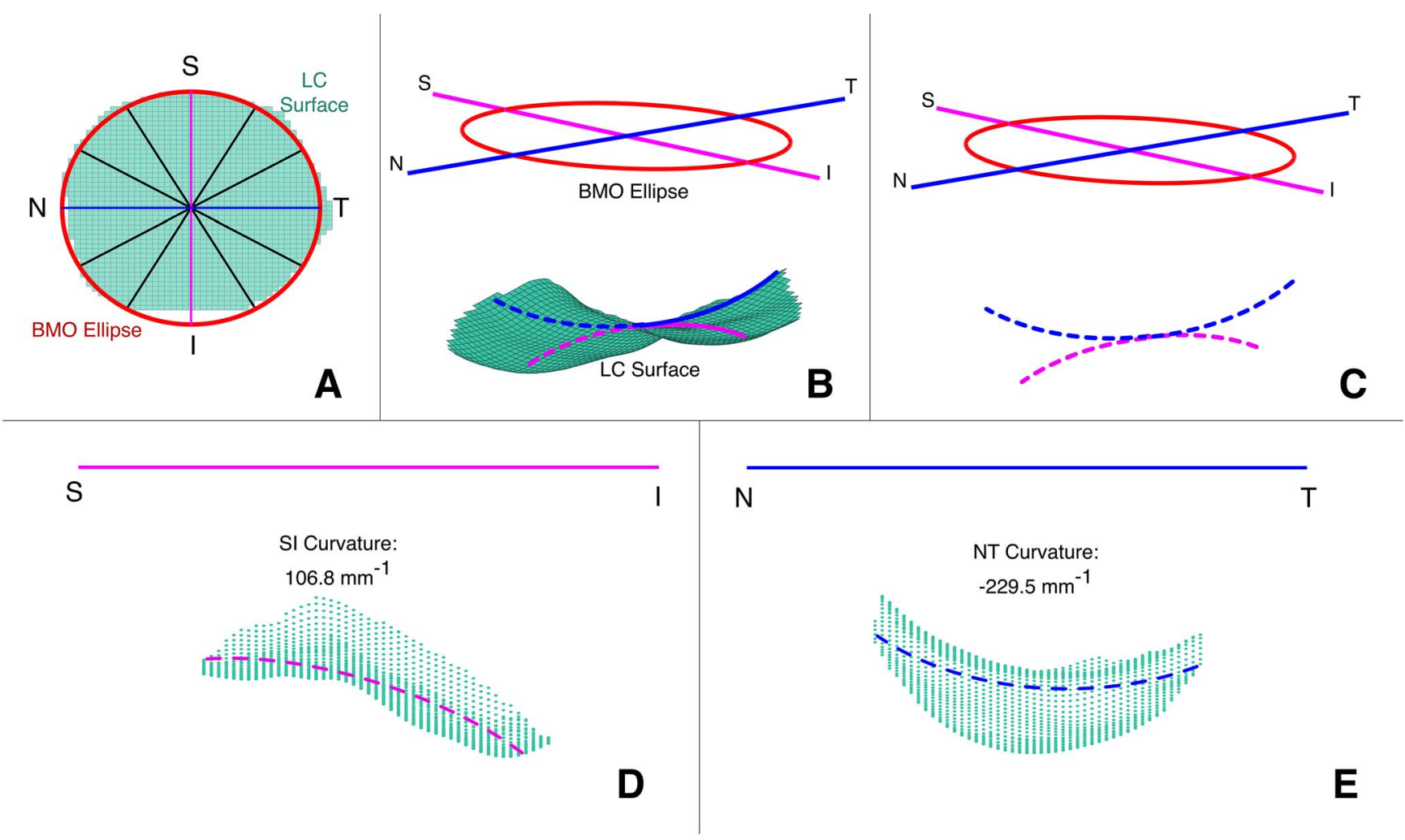

Figure 4. Estimation of LC curvature. (A) En face view depicting radial cross sections passing through the BMO center (in total, 180 evenly spaced cross sections were used); (B) calibrated LC (cyan) with BMO ellipse (red) with superior-inferior (pink) and nasal-temporal (blue) orientation lines; (C) best-fitting circular arc to LC along S-I (pink dotted) and N-T (blue dotted) directions; (D) projection along the S-I plane showing a positive arc curvature (anteriorly curved); (E) projection along the N-T plane showing a negative arc curvature (posteriorly curved).

(ICC) analysis was used to measure agreement. Additionally, the RMS error between the reconstructed LC surfaces generated from successive delineations of the same eye was calculated. Intra- and intervisit repeatability was performed on a set of 18 eyes that were scanned three times using OCT. The first two scans were performed during the same visit, and the third scan was performed on a second visit 1 week later. These scans were delineated by the same observer (SGT), and ICC analysis was performed.

\section{Statistical Analysis}

Linear regression analysis was used to identify associations of ocular and demographic factors with LC morphological parameters (LC-GSI, curvature, and depth). Factors with $P<$ 0.1 in the univariate analysis and biologically plausible association factors (sex, age, IOP, axial length, corneal curvature, CCT, BMO area, VCDR, and BMO-MRW) were included in the multivariate linear regression models. All

$\begin{array}{ccc}\text { GSI } & \text { Shape S-I Cross-section } \\ {[-7 / 8-5 / 8)} & \text { Trough } \\ {[-5 / 8-3 / 8)} & \text { Saddle rut } \\ {[-3 / 8-1 / 8)} & \text { Saddle }\end{array}$

FiguRE 5. LC-GSI, shape, and corresponding illustrative LC morphology along S-I and N-T cross sections; dashed lines denote illustrative LC morphology, and black curves denote the best-fitting arc. 


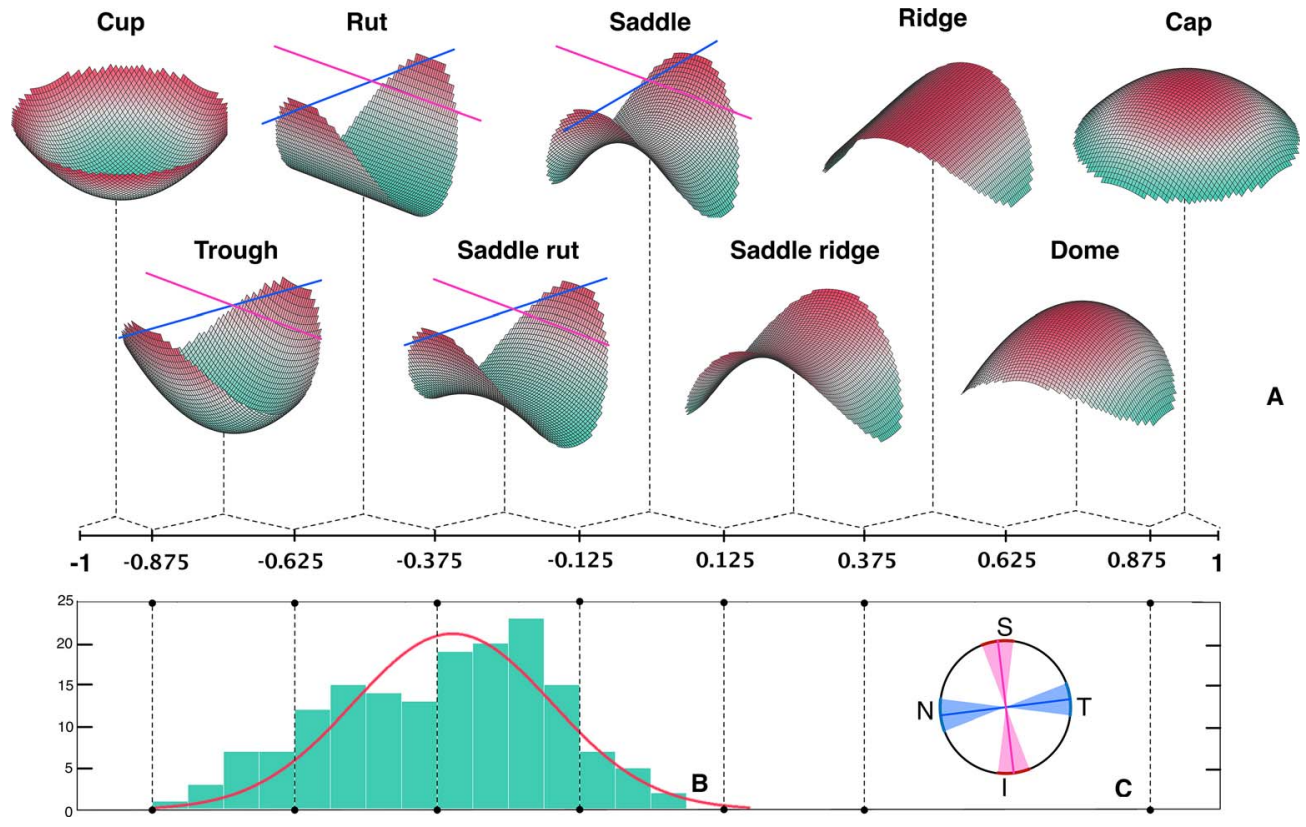

Figure 6. Visualization of global LC shape index: (A) The LC-GSI scale is divided into nine categories, and the corresponding predicted shapes from the center of each category are shown (with principal directions indicated by pink [max] and blue [min] lines); (B) the distribution of LC-GSI among healthy human subjects and a normal fit (red) with a mean of -0.34 and standard deviation 0.19 ; (C) en face view of the orientation of the principal directions in healthy human eyes (line indicates mean; wedge indicates standard deviation).

statistical analyses were carried out using Stata (v10.1; StataCorp LP, College Station, TX, USA).

\section{RESULTS}

Of the 223 subjects originally enrolled, 21 were excluded based on clinical exclusion criteria, and 40 eyes were excluded due to peripapillary atrophy (PPA) or poor LC visibility, leaving 162 eyes for analysis. The mean age of the included subjects was $58 \pm 7$ years, and 75 (46\%) of them were female. The demographics are shown in Table 1.

\section{Validation of Measurements}

The variations in arc curvature between actual LC surfaces and shapes predicted by LC-GSI are shown in Figure 7 . The normalized RMS error in arc curvature was found to be less than $8 \%$ for all cases.

Intraobserver repeatability $(n=50)$ showed good agreement, with an ICC greater than 0.93 for all measured parameters-LC-GSI, curvature, depth, and BMO-MRW. The average RMS error between repeated delineations was 8.96 $\mu \mathrm{m}$, which is approximately twice the axial resolution of the OCT scans $(3.9 \mu \mathrm{m})$. Intra- and intervisit repeatability $(n=18)$ showed fair to good repeatability, with an ICC greater than 0.82 for all measured parameters (Table 2).

\section{Distribution of LC Morphology}

The distribution of LC-GSI in healthy eyes is shown in Figure 6B. Of the 162 healthy eyes, 12 LCs (7\%) had a predicted shape falling under trough, 48 (30\%) under rut, 78 (48\%) under saddle rut, and 24 (15\%) under saddle. Moving from trough to saddle (increasing LC-GSI), the central ridge along the S-I cross section becomes more prominent and the LC is less posteriorly curved along the N-T cross section, especially toward the center (Fig. 5). Notably, no LCs had a cup-like or cap-like appearance. The average LC-GSI was $-0.34 \pm 0.19$. The principal directions (maximum and minimum arc curvatures) were consistently aligned along the S-I and N-T directions (with a spread of approximately $15^{\circ}$ ), respectively (Fig. 6C).

Consistent with the distribution of LC-GSI, the LC showed significant directional variation in arc curvature (Figs. 4D, 4E). Along the N-T cross section, arc curvature was $-340 \pm 116$ $\mathrm{m}^{-1}$, indicating an overall $\mathrm{U}$ shape. However, along the S-I cross section, arc curvature was $78 \pm 130 \mathrm{~m}^{-1}$, with the positive mean value indicating that the LC was typically curved

Table 1. Demographics of Study Subjects, $n=162$

\begin{tabular}{lcc}
\hline \multicolumn{1}{c}{ Characteristic } & Mean \pm SD & Range \\
\hline Sex, female (\%) & \multicolumn{2}{c}{$75(46.3 \%)$} \\
Age, $\mathrm{y}$ & $58 \pm 7$ & 48 to 84 \\
IOP, mm Hg & $15 \pm 2$ & 9 to 20 \\
Axial length, mm & $23.38 \pm 0.94$ & 21.53 to 27.57 \\
CCT, $\mu \mathrm{m}$ & $541 \pm 30$ & 443 to 617 \\
Corneal curvature, mm & $7.70 \pm 0.27$ & 7.01 to 8.34 \\
BMO area, mm ${ }^{2}$ & $2.29 \pm 0.42$ & 1.29 to 3.72 \\
VCDR & $0.41 \pm 0.1$ & 0.17 to 0.63 \\
LC depth, $\mu \mathrm{m}$ & & \\
$\quad$ Mean depth & $403 \pm 90$ & 215 to 689 \\
$\quad$ N-T depth & $388 \pm 92$ & 155 to 672 \\
$\quad$ S-I depth & $434 \pm 95$ & 239 to 715 \\
Curvature, m ${ }^{-1}$ & & \\
$\quad$ N-T curvature & $-340 \pm 116$ & -672 to -59 \\
$\quad$ S-I curvature & $78 \pm 130$ & -584 to +434 \\
Global shape index & $-0.34 \pm 0.19$ & -0.82 to +0.08 \\
Min. rim width, $\mu \mathrm{m}$ & $280 \pm 45$ & 162 to 419 \\
\hline
\end{tabular}

min., minimum. 

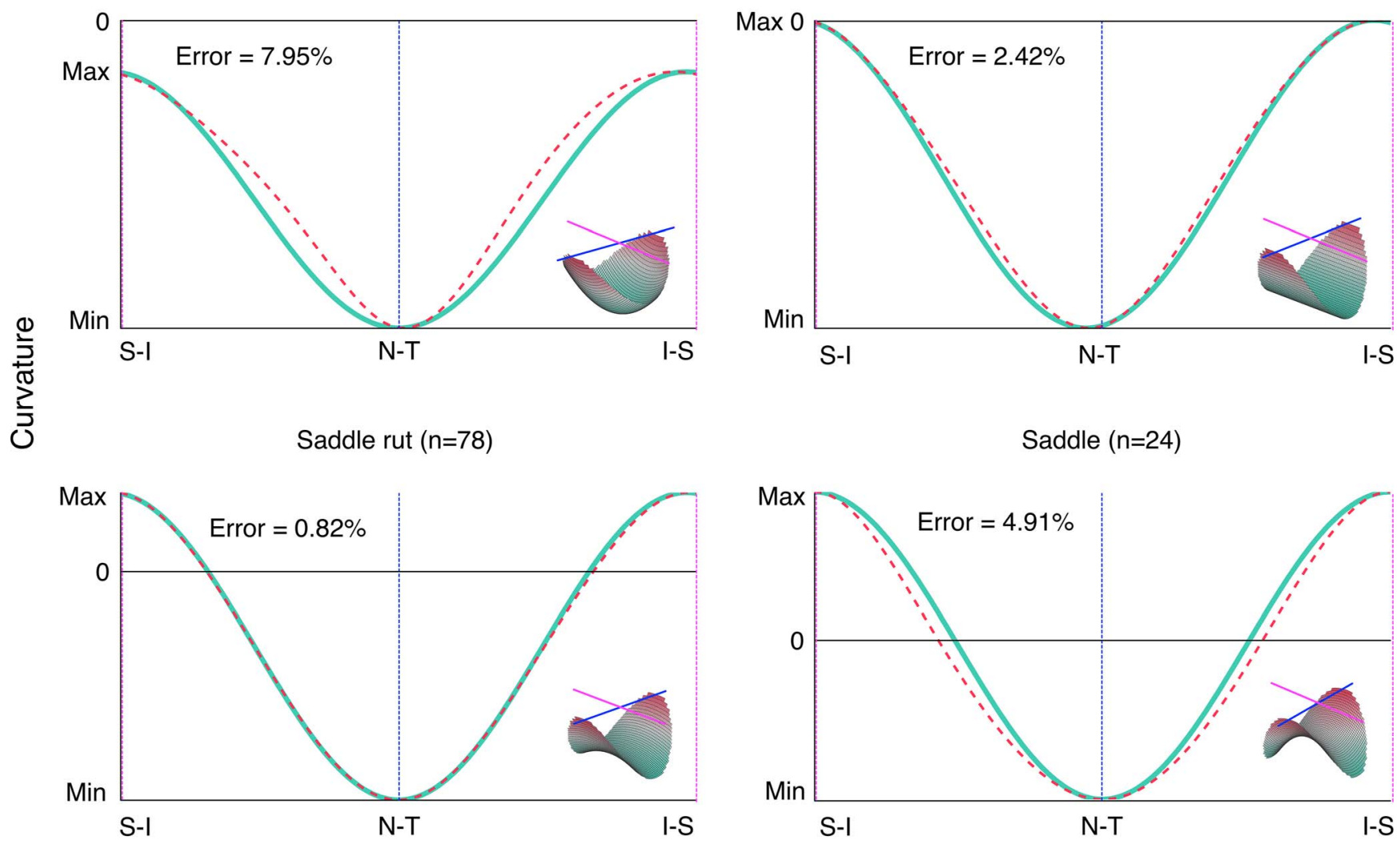

Orientation

FigurE 7. Validation of LC-GSI as a shape measure; actual LC arc curvature variations (cyan) are compared with curvature variations of predicted shapes (dotted pink) obtained using principal arc curvatures of the actual LCs; insets show predicted shapes.

anteriorly. The large standard deviation suggests that a posteriorly curved S-I cross section was not uncommon. The average shape of the LC along the S-I and N-T cross sections for the 162 eyes is shown in Figure 8.

Lamina cribrosa depth also showed significant regional variation. The LC was shallower along the N-T cross section (depth of $388 \pm 92 \mu \mathrm{m}$ ) when compared to the S-I cross section (depth of $434 \pm 95 \mu \mathrm{m}$ ). The LC insertion points were also deeper along the S-I cross section when compared to the $\mathrm{N}-\mathrm{T}$ cross section (Fig. 8). Mean depth for the entire LC was $403 \pm 90 \mu \mathrm{m}$.

\section{Determinants of LC Morphology}

In the multivariate regression analysis, axial length, BMO area, VCDR, and BMO-MRW were found to be significantly associated with LC-GSI (Table 3). A more trough-like LC (more negative LC-GSI) was associated with longer axial length $(\beta=$ $-0.067 ; P<0.001)$, larger BMO area $(\beta=-0.080 ; P=0.020)$, larger VCDR $(\beta=-0.04 ; P=0.007)$, and smaller BMO-MRW $(\beta$ $=0.0011 ; P<0.001$ ) (Fig. 9).

Lamina cribrosa depth, axial length, BMO area, VCDR, and BMO-MRW were found to be significantly associated with curvature. Increase in LC depth was associated with a decrease in N-T arc curvature (more posteriorly curved). Smaller axial length was associated with an increase in S-I arc curvature (more anteriorly curved). Smaller BMO area, smaller VCDR, and larger BMO-MRW were associated with both a decrease in $\mathrm{N}-\mathrm{T}$ arc curvature and an increase in S-I arc curvature. We found no significant associations between LC curvature and other ocular factors in the model, including age, IOP, and corneal curvature (Table 4).

Sex, axial length, VCDR, and BMO-MRW were significantly associated with mean LC depth. Specifically, increase in LC depth was associated with male sex $(\beta=53 ; P<0.001)$, smaller axial length $(\beta=-22 ; P=0.003)$, larger $\operatorname{VCDR}(\beta=26$; $P<0.001)$, and smaller BMO-MRW $(\beta=-0.44 ; P=0.002)$ (Fig.

TABLE 2. Assessment of Repeatability of Lamina Cribrosa Morphology Measurements

\begin{tabular}{lccc}
\hline & \multicolumn{2}{c}{ Intraclass Correlation Coefficient, ICC } \\
\cline { 2 - 4 } \multicolumn{1}{c}{ Parameter } & $\begin{array}{c}\text { Intraobserver, } \\
\boldsymbol{n}=\mathbf{5 0}\end{array}$ & $\begin{array}{c}\text { Intravisit, } \\
\boldsymbol{n}=\mathbf{1 8}\end{array}$ & $\begin{array}{c}\text { Intervisit, } \\
\boldsymbol{n}=\mathbf{1 8}\end{array}$ \\
\hline Global shape index & 0.952 & 0.901 & 0.900 \\
N-T curvature & 0.950 & 0.876 & 0.913 \\
S-I curvature & 0.937 & 0.821 & 0.844 \\
Mean LC depth & 0.995 & 0.983 & 0.990 \\
N-T depth & 0.993 & 0.984 & 0.992 \\
S-I depth & 0.995 & 0.995 & 0.993 \\
Min. rim width & 0.997 & 0.963 & 0.951 \\
\hline
\end{tabular}




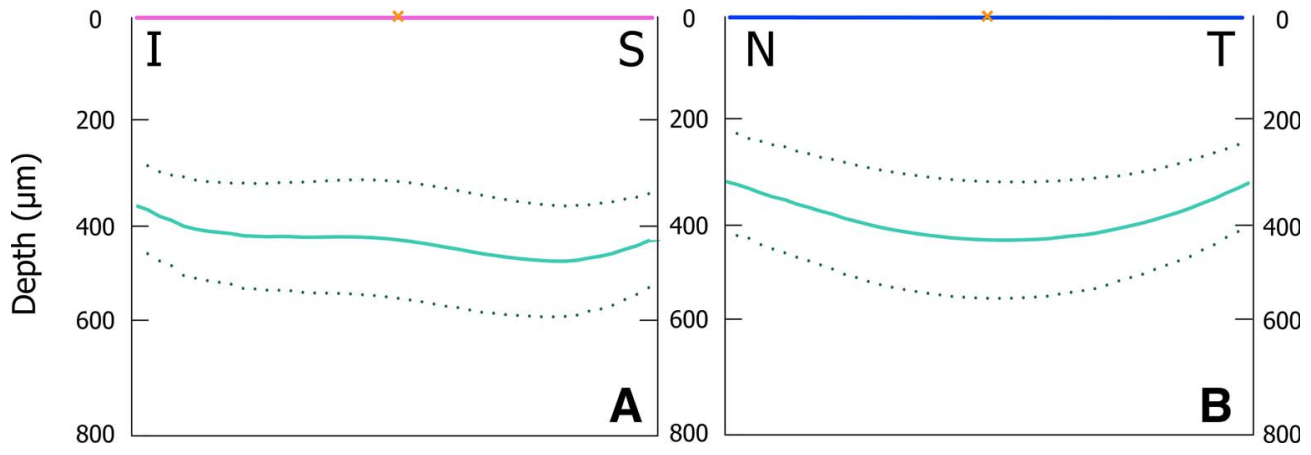

Figure 8. Mean depth and standard deviation of 162 LCs. (A) Along the superior-inferior direction, the insertion points are lower and the LC has a W shape; (B) along the nasal-temporal direction, the insertion points are higher and the LC has a U shape.

10). Notably, age and IOP (within normal IOP range examined) were not significantly associated with LC depth (Table 5). The associations remained the same with LC depth along the N-T and S-I directions.

\section{Discussion}

In this study, we introduced a new morphological parameter, LC-GSI, to characterize anterior LC shape. The LC-GSI offers several novelties. First, it specifies the global geometry of a surface, ${ }^{37}$ which provides additional insight into LC morphology compared to the current measurement of LC depth. Second, since LC-GSI is a measure of shape and not position, it is independent of the BMO or other reference structures, removing any measurement bias that may arise due to variations in the region surrounding the LC. ${ }^{20}$ Third, it provides a single metric that is intuitive and easy to visualize-an essential requirement for clinical translation. Taken together, LC depth and LC-GSI quantify position as well as shape, providing a more holistic measure of LC morphology.

\section{Distribution and Determinants of LC-GSI}

The distribution of LC-GSI in healthy eyes ranged from trough to saddle, with saddle rut being the most common (Fig. 6B). As we move from trough to saddle (increasing LC-GSI), the LC becomes less posteriorly curved along the N-T direction and the central ridge becomes increasingly more prominent in S-I

Table 3. Multivariate Linear Regression Assessing the Associations With Global Shape Index (LC-GSI) of the Anterior Lamina Cribrosa Surface, $n=162$

\begin{tabular}{lllr}
\hline \multicolumn{1}{c}{ Determinant } & \multicolumn{1}{c}{$\boldsymbol{\beta}$} & \multicolumn{1}{c}{ 95\% CI } & $\boldsymbol{P}$ Value \\
\hline LC depth, $\mu \mathrm{m}$ & -0.0002 & -0.0006 to 0.00004 & 0.092 \\
Male sex & -0.032 & -0.09 to 0.03 & 0.288 \\
Age, $\mathrm{y}$ & -0.0018 & -0.006 to 0.002 & 0.338 \\
IOP, mm Hg & -0.0014 & -0.014 to 0.011 & 0.818 \\
Axial length, mm & -0.067 & -0.097 to -0.037 & $<0.001$ \\
CCT, $\mu \mathrm{m}$ & -0.0004 & -0.001 to 0.0005 & 0.378 \\
CC, mm & -0.04 & -0.088 to 0.16 & 0.532 \\
BMO area, mm ${ }^{2}$ & -0.08 & -0.15 to -0.012 & 0.020 \\
VCDR $^{*}$ (per 0.1 increase) & -0.04 & -0.07 to -0.01 & 0.007 \\
Min. rim width, $\dagger \mu \mathrm{m}$ & 0.0011 & 0.0005 to 0.00170 & 0.001
\end{tabular}

CI, confidence interval; $\mathrm{CC}$, corneal curvature.

* When VCDR was included in the model, BMO area and min. rim width were excluded since they are highly correlated.

t When min. rim width was included in the model, BMO area and VCDR were excluded. cross sections (Fig. 5). Previous studies have reported the LC to have a central ridge ${ }^{13,14}$ and a corresponding $U$ or $W$ shape. ${ }^{5,13}$ Using LC-GSI, we are for the first time able to provide a quantitative assessment of the prominence of this central ridge and the corresponding global LC shape.

In healthy eyes, the cross sections that contain the principal LC arc curvatures are roughly perpendicular and aligned to the $\mathrm{N}-\mathrm{T}$ and S-I directions (Fig. 6C). Existing in vivo studies on LC morphology using OCT vary in their scanning methodologies, ranging from several radial scans ${ }^{7,10}$ to just a few horizontal or vertical scans. ${ }^{5,13,14}$ Since the principal directions appear to be aligned along the N-T and S-I cross sections, our finding suggests that scans along these two cross sections alone may be sufficient in calculating LC-GSI and estimating anterior LC shape. A comparison of the original LC-GSI values computed using the entire reconstructed LC and a new estimate based on just the N-T and S-I cross sections showed very good agreement (Pearson's correlation $R=0.92$ ). This possibility of estimating LC-GSI from just two OCT B-scans could potentially offer a quick and straightforward clinical assessment of anterior LC shape.

Larger axial length, BMO area, and VCDR, as well as smaller BMO-MRW, are significantly associated with a decrease in LCGSI (movement from saddle to trough, as shown in Fig. 9). Vertical cup-to-disc ratio and BMO-MRW are measures of optic disc cupping, and since the LC lies right below the optic cup, the association between optic disc cupping and deviation of

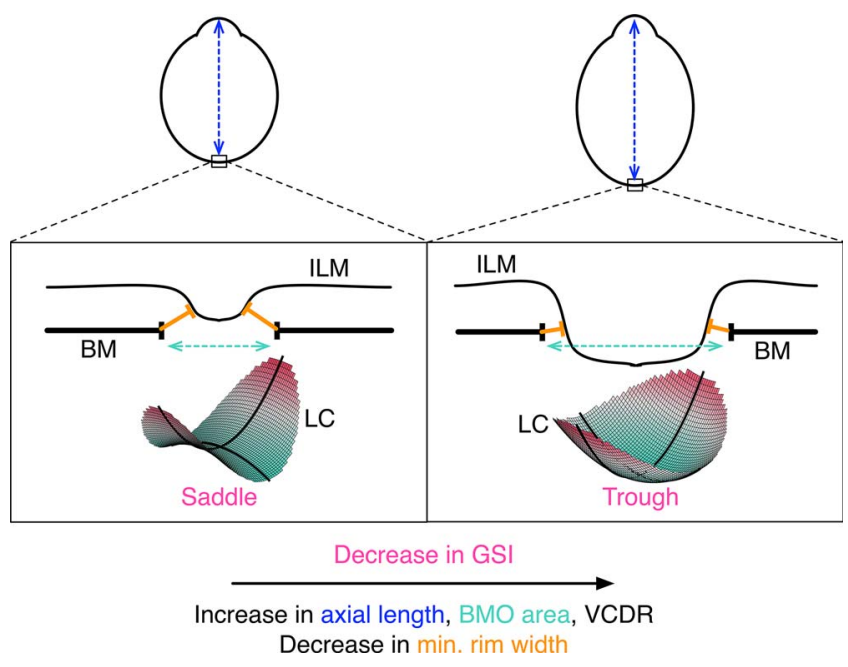

Figure 9. Illustration of the determinants of LC-GSI in healthy eyes (not to scale); a decrease in LC-GSI is associated with larger axial length, larger BMO area, larger VCDR, and a smaller BMO-MRW. 
TABLE 4. Multivariate Linear Regression Assessing the Associations With Curvature of the Anterior Lamina Cribrosa Surface, $n=162$

\begin{tabular}{|c|c|c|c|c|c|c|}
\hline \multirow[b]{2}{*}{ Determinant } & \multicolumn{3}{|c|}{ Curvature N-T } & \multicolumn{3}{|c|}{ Curvature S-I } \\
\hline & $\beta$ & $95 \% \mathrm{CI}$ & $P$ Value & $\beta$ & $95 \% \mathrm{CI}$ & $P$ Value \\
\hline LC depth, $\mu \mathrm{m}$ & -0.8 & -0.9 to -0.6 & $<0.001$ & 0.1 & -0.2 to 0.3 & 0.629 \\
\hline Male sex & 7.4 & -24 to 39 & 0.647 & -14 & -57 to 30 & 0.538 \\
\hline Age, y & 0.5 & -1.5 to 2.5 & 0.616 & -1.5 & -4.2 to 1.3 & 0.29 \\
\hline IOP, $\mathrm{mm} \mathrm{Hg}$ & -3.0 & -9.4 to 3.4 & 0.354 & -1.4 & -10.1 to 7.4 & 0.762 \\
\hline Axial length, mm & -11 & -32 to 9 & 0.286 & -40 & -68 to -11 & 0.006 \\
\hline $\mathrm{CCT}, \mu \mathrm{m}$ & -0.2 & -0.7 to 0.3 & 0.464 & 0.0 & -0.7 to 0.6 & 0.919 \\
\hline $\mathrm{CC}, \mathrm{mm}$ & -5.8 & -74 to 62 & 0.867 & -1.1 & -94 to 91 & 0.981 \\
\hline $\mathrm{BMO}$ area, $\mathrm{mm}^{2}$ & 55 & 20 to 90 & 0.002 & -62 & -110 to -14 & 0.012 \\
\hline $\mathrm{VCDR}^{*}$ (per 0.1 increase) & 29 & 13 to 45 & $<0.001$ & -35 & -56 to -13 & 0.002 \\
\hline Min. rim width, $\dagger \mu \mathrm{m}$ & -0.8 & -1.1 to -0.5 & $<0.001$ & 0.75 & 0.29 to 1.21 & 0.001 \\
\hline
\end{tabular}

* When VCDR was included in the model, BMO area and min. rim width were excluded since they are highly correlated.

† When min. rim width was included in the model, BMO area and VCDR were excluded.

the LC from a saddle shape indicates that prelaminar optic disc changes could be associated with underlying changes in LC morphology. Since large axial length, optic disc size, and VCDR are all risk factors for glaucoma, ${ }^{38,39} \mathrm{LC}$ deviation from a saddle shape may be indicative of pathology or represent a risk factor. Further investigation is necessary to establish a causal link.

\section{Distribution and Determinants of LC Depth}

We found the LC to be significantly deeper along the S-I direction when compared to the N-T direction. Early studies have shown that there is a greater density of connective tissue and glial cells in the nasal and temporal quadrants when compared to the superior and inferior quadrants. ${ }^{17}$ This difference in supporting tissue may explain the difference in LC depth.

Male sex, smaller axial length, smaller BMO-MRW, and larger VCDR were significantly associated with a deeper LC. The direction of association of LC depth with sex and axial length is consistent with a previous finding. ${ }^{14}$ The association of LC depth with BMO-MRW and VCDR in healthy eyes has not been reported previously. Bruch's membrane opening-MRW has been shown to be more accurate than neuroretinal rim width measured from fundus images. ${ }^{36}$ Furthermore, thinning of the rim has been shown to precede RNFL changes during

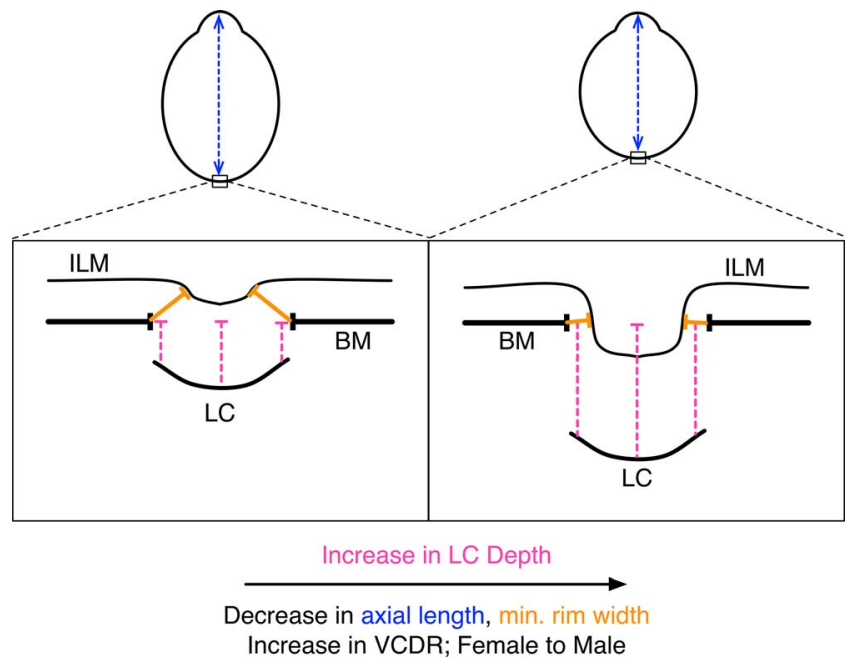

Figure 10. Illustration of the determinants of LC depth in healthy eyes (not to scale); an increase in LC depth is associated with smaller axial length, smaller BMO-MRW, larger VCDR, and male sex. glaucoma onset. ${ }^{10}$ The association of LC depth with BMOMRW and VCDR, both of which measure prelaminar ONH changes, further suggests a link between prelaminar optic disc changes and LC morphology.

We found that age, corneal curvature, CCT, and IOP were not associated with LC depth. Whereas no studies have shown an association between LC depth and corneal curvature, CCT, or IOP in a healthy population, findings on its association with age are conflicting. Seo et al. ${ }^{14}$ found no association in a Korean population, whereas Rhodes et al. ${ }^{40}$ found a significant association among people of European and African ancestry. Interestingly, Rhodes et al. ${ }^{40}$ reported a negative association among European descendants and a positive association among African descendants. Since our study population was exclusively Indian, differences between these findings may be explained by differing ethnicities of the study groups. The observed nil association may also be explained by variations in the axial position of the BMO. Specifically, a previous study showed that the BMO migrated posteriorly with age. ${ }^{20}$ Considering that the BMO was used as a reference in our measurement of LC depth, age-related posterior migration of the BMO might potentially confound the relationship between age and LC depth, thus resulting in the observed nil association. A possible reason for not finding an association between LC depth and IOP may be that subjects with IOP greater than $21 \mathrm{~mm} \mathrm{Hg}$ were classified as glaucoma suspect and excluded from analysis in our study.

Table 5. Multivariate Linear Regression Assessing the Associations With Depth of the Anterior Lamina Cribrosa Surface, $n=162$

\begin{tabular}{lccr}
\hline & \multicolumn{3}{c}{ Mean Depth } \\
\cline { 2 - 4 } \multicolumn{1}{c}{ Determinant } & $\boldsymbol{\beta}$ & $\mathbf{9 5 \%}$ CI & $\boldsymbol{P}$ Value \\
\hline Male sex & 53 & 27 to 79 & $<0.001$ \\
Age, $\mathrm{y}$ & -0.9 & -2.7 to 0.9 & 0.307 \\
IOP, mm Hg & 1.5 & -4.2 to 7.1 & 0.615 \\
Axial length, mm & -22 & -36 to -7 & 0.003 \\
CCT, $\mu \mathrm{m}$ & -0.1 & -0.5 to 0.3 & 0.642 \\
CC, mm & -17 & -73 to 40 & 0.564 \\
BMO area, mm ${ }^{2}$ & -22 & -51 to 8 & 0.148 \\
VCDR $^{*}$ (per 0.1 increase) & 26 & 13 to 39 & $<0.001$ \\
Min. rim width, $\dagger \mu \mathrm{m}$ & -0.44 & -0.73 to -0.16 & 0.002 \\
\hline
\end{tabular}

* When VCDR was included in the model, BMO area and min. rim width were excluded since they are highly correlated.

t When min. rim width was included in the model, BMO area and VCDR were excluded. 


\section{Limitations}

Restricted LC visibility in SD-OCT poses some limitations on our methodology. In spite of demonstrating good repeatability, small local variations in delineation of $\mathrm{ONH}$ structures exist and become especially significant in a second-order characterization of shape, such as measurement of curvature and LC-GSI. To minimize the effect of this variation, we estimated arc curvature across entire cross sections and introduced a global shape index (as opposed to a local measure). The cost of making our measurements more robust is that they may be less sensitive to local LC shape changes such as focal defects. However, since there is currently no generally accepted parameter that both is robust and gives a localized estimate of LC, our novel characterization using LCGSI provides a quantitative and objective measure of LC shape. Our measurements of curvature were better able to capture a simpler U shape along N-T cross sections of the LC than a more complex W shape along S-I cross sections (Fig. 5). Better LC visibility due to future improvements in imaging modalities may allow us to measure curvature locally and detect local LC shape changes without compromising on robustness.

Finally, this study included only healthy subjects. The distribution of ocular parameters, particularly IOP and VCDR, may be different among healthy and glaucoma eyes. In order to better understand LC shape changes during glaucoma onset, the healthy controls will have to be compared with glaucoma patients.

In conclusion, in this study we examined LC shape in healthy human eyes using a new morphological parameter, LCGSI. This single index is able to quantitatively characterize global anterior LC morphology and is intuitive to visualize. It therefore has strong potential for clinical translation. We also identified that an increase in axial length, BMO area, and VCDR and decrease in BMO-MRW were associated with a LC that has a less prominent central ridge (decrease in LC-GSI). Our measurements of LC depth and its determinants were consistent with previous findings.

\section{Acknowledgments}

Supported by a National University of Singapore (NUS) CrossFaculty Grant (R-397-000-176-133), the Ministry of Education, Academic Research Funds, Tier 1 (R-397-000-140-133), and a NUS Young Investigator Award (NUSYIA_FY13_P03, R-397-000-174133) (MJAG); the National Medical Research Council Singapore (CSA/033/2012) (C-YC); and in part by the United Kingdom Department of Health through the award made by the National Institute for Health Research to Moorfields Eye Hospital National Health Service Foundation Trust and University College London Institute of Ophthalmology for a Biomedical Research Centre for Ophthalmology (NGS). The views expressed in this publication are those of the authors and not necessarily those of the United Kingdom Department of Health.

Disclosure: S.G. Thakku, None; Y.-C. Tham, None; M. Baskaran, None; J.-M. Mari, None; N.G. Strouthidis, None; T. Aung, None; C.-Y. Cheng, None; M.J.A. Girard, None

\section{References}

1. Quigley HA. Open-angle glaucoma. New Engl J Med. 1993; 328:1097-1106.

2. Quigley HA, Addicks EM, Green WR. Optic nerve damage in human glaucoma. III. Quantitative correlation of nerve fiber loss and visual field defect in glaucoma, ischemic neuropathy, papilledema, and toxic neuropathy. Arch Ophthalmol. 1982; 100:135-146.
3. Quigley HA, Addicks EM, Green WR, Maumenee AE. Optic nerve damage in human glaucoma. II. The site of injury and susceptibility to damage. Arch Ophthalmol. 1981;99:635-649.

4. Downs JC, Roberts MD, Burgoyne CF. Mechanical environment of the optic nerve head in glaucoma. Optom Vis Sci. 2008;85: 425-435.

5. Furlanetto RL, Park SC, Damle UJ, et al. Posterior displacement of the lamina cribrosa in glaucoma: in vivo interindividual and intereye comparisons. Invest Ophthalmol Vis Sci. 2013;54: 4836-4842.

6. Jung KI, Jung Y, Park KT, Park CK. Factors affecting plastic lamina cribrosa displacement in glaucoma patients. Invest Ophthalmol Vis Sci. 2014;55:7709-7715.

7. Ren R, Yang H, Gardiner SK, et al. Anterior lamina cribrosa surface depth, age, and visual field sensitivity in the Portland Progression Project. Invest Ophthalmol Vis Sci. 2014;55: 1531-1539.

8. Crawford Downs J, Roberts MD, Sigal IA. Glaucomatous cupping of the lamina cribrosa: a review of the evidence for active progressive remodeling as a mechanism. Exp Eye Res. 2011;93:133-140.

9. Bellezza AJ, Rintalan CJ, Thompson HW, Downs JC, Hart RT, Burgoyne CF. Deformation of the lamina cribrosa and anterior scleral canal wall in early experimental glaucoma. Invest Ophthalmol Vis Sci. 2003;44:623-637.

10. Strouthidis NG, Fortune B, Yang H, Sigal IA, Burgoyne CF. Longitudinal change detected by spectral domain optical coherence tomography in the optic nerve head and peripapillary retina in experimental glaucoma. Invest Ophthalmol Vis Sci. 2011;52:1206-1219.

11. Yang H, Williams G, Downs JC, et al. Posterior (outward) migration of the lamina cribrosa and early cupping in monkey experimental glaucoma. Invest Ophthalmol Vis Sci. 2011;52: 7109-7121.

12. Yang H, Downs JC, Bellezza A, Thompson H, Burgoyne CF. 3-D histomorphometry of the normal and early glaucomatous monkey optic nerve head: prelaminar neural tissues and cupping. Invest Ophthalmol Vis Sci. 2007;48:5068-5084.

13. Park SC, Kiumehr S, Teng CC, Tello C, Liebmann JM, Ritch R. Horizontal central ridge of the lamina cribrosa and regional differences in laminar insertion in healthy subjects. Invest Ophthalmol Vis Sci. 2012;53:1610-1616.

14. Seo JH, Kim TW, Weinreb RN. Lamina cribrosa depth in healthy eyes. Invest Ophthalmol Vis Sci. 2014;55:1241-1251.

15. Burgoyne CF, Downs JC, Bellezza AJ, Hart RT. Threedimensional reconstruction of normal and early glaucoma monkey optic nerve head connective tissues. Invest Ophthalmol Vis Sci. 2004;45:4388-4399.

16. Dandona L, Quigley HA, Brown AE, Enger C. Quantitative regional structure of the normal human lamina cribrosa. A racial comparison. Arch Ophthalmol. 1990;108:393-398.

17. Radius RL, Gonzales M. Anatomy of the lamina cribrosa in human eyes. Arch Ophthalmol. 1981;99:2159-2162.

18. Downs JC, Yang H, Girkin C, et al. Three-dimensional histomorphometry of the normal and early glaucomatous monkey optic nerve head: neural canal and subarachnoid space architecture. Invest Ophthalmol Vis Sci. 2007;48:31953208.

19. Yang H, Downs JC, Girkin C, et al. 3-D histomorphometry of the normal and early glaucomatous monkey optic nerve head: lamina cribrosa and peripapillary scleral position and thickness. Invest Ophthalmol Vis Sci. 2007;48:4597-4607.

20. Johnstone J, Fazio M, Rojananuangnit $\mathrm{K}$, et al. Variation of the axial location of Bruch's membrane opening with age, choroidal thickness, and race. Invest Ophthalmol Vis Sci. 2014;55:2004-2009. 
21. Sanfilippo PG, Grimm JL, Flanagan JG, Lathrop KL, Sigal IA. Application of Elliptic Fourier analysis to describe the lamina cribrosa shape with age and intraocular pressure. Exp Eye Res. 2014;128:1-7.

22. Sredar N, Ivers KM, Queener HM, Zouridakis G, Porter J. 3D modeling to characterize lamina cribrosa surface and pore geometries using in vivo images from normal and glaucomatous eyes. Biomed Opt Express. 2013;4:1153-1165.

23. Lavanya R, Jeganathan VS, Zheng Y, et al. Methodology of the Singapore Indian Chinese Cohort (SICC) eye study: quantifying ethnic variations in the epidemiology of eye diseases in Asians. Ophthalmic Epidemiol. 2009;16:325-336.

24. Spaide RF, Koizumi H, Pozzoni MC. Enhanced depth imaging spectral-domain optical coherence tomography. Am J Ophthalmol. 2008;146:496-500.

25. Lee EJ, Kim TW, Weinreb RN, Park KH, Kim SH, Kim DM. Visualization of the lamina cribrosa using enhanced depth imaging spectral-domain optical coherence tomography. Am J Ophthalmol. 2011;152:87-95, e81.

26. Sharma U, Chang EW, Yun SH. Long-wavelength optical coherence tomography at 1.7 microm for enhanced imaging depth. Opt Express. 2008;16:19712-19723.

27. Spaide RF. Enhanced depth imaging optical coherence tomography of retinal pigment epithelial detachment in agerelated macular degeneration. Am J Ophthalmol. 2009;147: 644-652.

28. Sigal IA, Wang B, Strouthidis NG, Akagi T, Girard MJ. Recent advances in OCT imaging of the lamina cribrosa. $\mathrm{Br} J$ Ophthalmol. 2014;98(suppl 2):ii34-ii39.

29. Girard MJ, Strouthidis NG, Ethier CR, Mari JM. Shadow removal and contrast enhancement in optical coherence tomography images of the human optic nerve head. Invest Ophthalmol Vis Sci. 2011;52:7738-7748.

30. Mari JM, Strouthidis NG, Park SC, Girard MJ. Enhancement of lamina cribrosa visibility in optical coherence tomography images using adaptive compensation. Invest Ophthalmol Vis Sci. 2013;54:2238-2247.

31. Gupta P, Sidhartha E, Girard MJ, Mari JM, Wong TY, Cheng CY. A simplified method to measure choroidal thickness using adaptive compensation in enhanced depth imaging optical coherence tomography. PLoS One. 2014;9:e96661.

32. Girard MJ, Tun TA, Husain R, et al. Lamina cribrosa visibility using optical coherence tomography imaging: comparison of devices and effects of enhanced depth imaging and adaptive compensation. Invest Ophthalmol Vis Sci. 2015. In press.

33. Strouthidis NG, Grimm J, Williams GA, Cull GA, Wilson DJ, Burgoyne CF. A comparison of optic nerve head morphology viewed by spectral domain optical coherence tomography and by serial histology. Invest Ophthalmol Vis Sci. 2010;51:14641474.

34. Koenderink JJ, Vandoorn AJ. Surface shape and curvature scales. Image Vis Comput. 1992;10:557-564.

35. Chauhan BC, O'Leary N, Almobarak FA, et al. Enhanced detection of open-angle glaucoma with an anatomically accurate optical coherence tomography-derived neuroretinal rim parameter. Ophthalmology. 2013;120:535-543.

36. Gardiner SK, Ren R, Yang H, Fortune B, Burgoyne CF, Demirel $\mathrm{S}$. A method to estimate the amount of neuroretinal rim tissue in glaucoma: comparison with current methods for measuring rim area. Am J Ophthalmol. 2014;157:540-549, e541-e542.

37. Kreyszig E. Differential Geometry. 1st ed. New York: Dover; 1991:384.

38. Hoffmann EM, Zangwill LM, Crowston JG, Weinreb RN. Optic disk size and glaucoma. Surv Ophthalmol. 2007;52:32-49.

39. Oku Y, Oku H, Park M, et al. Long axial length as risk factor for normal tension glaucoma. Graefes Arch Clin Exp Ophthalmol. 2009;247:781-787.

40. Rhodes LA, Huisingh C, Johnstone J, et al. Variation of laminar depth in normal eyes with age and race. Invest Ophthalmol Vis Sci. 2014;55:8123-8133. 